\title{
Radiation Effect on Unsteady Mixed Convection Boundary Layer Flow, Heat and Mass Transfer over a Wedge
}

\author{
N. C. Roy", M. N. Firoza and A. K. Halder \\ Department of Mathematics, Dhaka University, Dhaka-1000, Bangladesh
}

(Received: 9 July 2015; Accepted: 15 December 2015)

\begin{abstract}
This paper concerns the unsteady mixed convection laminar boundary layer flow past a vertical wedge in the presence of thermal radiation. The governing equations have been solved by the straightforward finite difference method for the entire frequency range. We observe that the Richardson's number, $R i$, strongly affects the skin friction, heat transfer and mass transfer. The effect of the Schmidt number, $S c$, on the mass transfer is significant, whereas the skin friction and the heat transfer are almost unaffected by it. Also the heat transfer is considerably dependent on the conduction-radiation parameter, $R_{d}$, but the influence of this parameter on the skin friction and the mass transfer is rather weak
\end{abstract}

\section{Introduction}

Mixed convection has been the focus of research because of its application in electronic equipment cooled by a fan and flows in the ocean and in the atmosphere. In this phenomena, the forced and the free convection effects are of comparable magnitude. Therefore, mixed convection takes place when the effect of buoyancy forces on a forced flow or vice versa is significant. There are various applications in thermal engineering and science where the influence of mixed convection is to be investigated. Examples of these applications include atmospheric boundary layer flows, solar energy systems, boilers, compact heat exchangers, and cooling of electronic devices.

Due to the occurrence in many interesting and important fluid-mechanical problems, oscillating flow and heat transfer under the influence of free-stream oscillation have been the important topic of research, for example, the accelerating and decelerating phases of missile flight, the intermittent flow in an engine during unstable combustion, heat transfer encountered in liquid rocket and turbo-jet engines, and thermal failure of the resonance tube heating in which the effect of heat generation appears to be significant. Lighthill $^{1}$ first initiated the investigation of how a boundary layer responds to fluctuations of the external velocity about a steady mean. Yih ${ }^{2}$ presented an analysis of the forced convection boundary-layer flow over a wedge with uniform suction and blowing, whereas Watanabe ${ }^{3}$ investigated the behavior of the boundary-layer over a wedge with suction and injection in forced flow. Recently, Sing et al. ${ }^{4}$ have studied the behavior of unsteady mixed convection flow of an incompressible viscous fluid over a vertical wedge with constant suction/injection. Al-Harbi and Ibrahim 5 investigated the development of mixed-convection boundary-layer flow along a symmetric wedge with variable surface temperature embedded in a saturated porous medium. Prasad et al. ${ }^{6}$ analyzed the magnetohydrodynamics mixed convection flow over a permeable non-isothermal wedge in the presence of variable thermal conductivity. A steady mixed convection boundary layer flow over a vertical wedge with the effect of magnetic field embedded in a porous medium has been studied by Kumari et al. ${ }^{7}$. Hossain et al. ${ }^{8}$ examined a steady two dimensional laminar forced flow of a viscous incompressible fluid past a horizontal wedge with uniform surface heat flux. Moreover, Kandasamy et al. ${ }^{9}$ presented the effects of variable viscosity and thermophoresis on magneto-hydrodynamics mixed convective heat and mass transfer past a porous wedge in the presence of chemical reaction.

The radiative effects have important applications in physics and engineering particularly in space technology and high temperature processes such as nuclear power plants, gas turbines and thermal energy storage. Yih ${ }^{10}$ studied the effect of radiation on mixed convection flow optically dense viscous fluids about an isothermal wedge embedded in a saturated porous medium. Chamkha et al. ${ }^{11}$ investigated the steadystate, hydromagnetic forced convective boundary-layer flow of an incompressible Newtonian, electrically conducting and heat-generating/absorbing fluid over a non-isothermal wedge in the presence of thermal radiation effects.

Many transport processes occurring both in nature and in industries involve fluid flow with the combined heat and mass transfer. Coupled heat and mass transfer problems in presence of thermal radiation are of importance in many processes and have, therefore, received considerable attention in recent times. The study of heat and mass transfer with thermal radiation is of great practical importance to engineers and scientists because of its almost universal occurrence in many branches of science and engineering. Motivated by the aforementioned applications, the present analysis is devoted to study the unsteady laminar boundary layer mixed convective heat and mass transfer with conduction-radiation interaction of viscous incompressible fluid past a vertical wedge.

\section{Mathematical Formulation}

A two-dimensional unsteady laminar mixed convection boundary layer flow of an optically dense viscous and incompressible fluid past a wedge under the influence of thermal radiation is considered. The physical configuration and coordinate system of the problem are shown in Figure 1. In this figure, $\delta_{M}, \delta_{T}$ and $\delta_{C}$ represent the approximate momentum, thermal and concentration boundary layer thickness, respectively. It is assumed that the surface temperature of the wedge, species concentration and free stream are oscillating with small amplitude but not in the direction.

*Author for correspondence. e-mail: nepal@du.ac.bd 
Under the usual Boussinesq approximation, the conservation equations for the unsteady, laminar, two dimensional boundary layer flow problem can be written as

$\frac{\partial u}{\partial x}+\frac{\partial v}{\partial y}=0$

$\frac{\partial u}{\partial t}+u \frac{\partial u}{\partial x}+v \frac{\partial u}{\partial y}=\frac{\partial U}{\partial t}+U \frac{\partial U}{\partial x}+v \frac{\partial^{2} u}{\partial y^{2}}$

$+g\left\{\beta_{T}\left(T-T_{\infty}\right)+\beta_{C}\left(C-C_{\infty}\right)\right\} \cos (\pi / 4)$,

$\frac{\partial T}{\partial t}+u \frac{\partial T}{\partial x}+v \frac{\partial T}{\partial y}=\alpha\left(\frac{\partial^{2} T}{\partial y^{2}}-\frac{1}{\kappa} \frac{\partial q_{r}}{\partial y}\right)$,

$\frac{\partial C}{\partial t}+u \frac{\partial C}{\partial x}+v \frac{\partial C}{\partial y}=D \frac{\partial^{2} C}{\partial y^{2}}$.

In the above equations, $x$ and $y$ are the coordinates parallel with and perpendicular to the wedge surface, $u$ and $v$ are the velocity components in the $x$ - and $y$-directions, respectively, $v$ is the coefficient of viscosity, $g$ is the acceleration due to gravity, $\beta_{T}$ and $\beta_{C}$ are the coefficient of volumetric expansion for temperature and concentration, respectively, $\alpha$ is the thermal diffusivity, $\kappa$ is the thermal conductivity and $D$ is the molecular diffusivity of the species. Furthermore, $T$ and $T_{\infty}$ are the temperature of the fluid in the boundary layer and the ambient fluid, respectively, $C$ and $C_{\infty}$ are the species concentration and the ambient concentration.

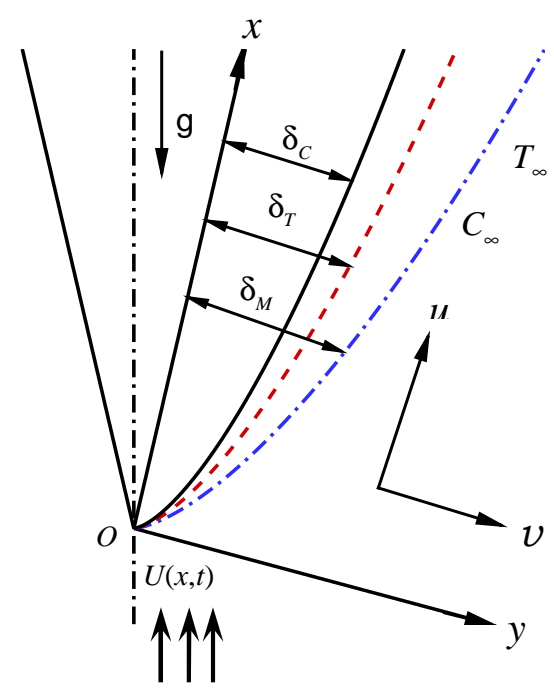

Fig. 1. Flow configuration and coordinate system

The corresponding boundary conditions are

$$
\begin{aligned}
& u=v=0, T=T_{\infty}+\left(T_{w}-T_{\infty}\right)\left(1+\varepsilon e^{i \omega t}\right), \\
& C=C_{\infty}+\left(C_{w}-C_{\infty}\right)\left(1+\varepsilon e^{i \omega t}\right) \text { at } y=0 \\
& u=U(x, t), T=T_{\infty}, C=C_{\infty} \text { as } y \rightarrow \infty .
\end{aligned}
$$

Here, $T_{w}$ is the mean surface temperature wherever $T_{w}>T_{\infty}$ and $C_{w}$ is the mean surface concentration with $C_{w}>C_{\infty}$. In addition the free-stream velocity $U(x, t)$ is assumed to be of the form

$U(x, t)=U_{0} x^{1 / 2}\left(1+\varepsilon e^{i \omega t}\right)$,

where $U_{0}, \varepsilon$ are constants and $\omega$ is the frequency.

The quantity $q_{r}$ on the right-hand side of equation (3) represents the radiative heat-flux in the $y$-direction. In order to reduce the complexity of the problem the optically thick radiation limit is considered in the present analysis. Thus the radiative heat-flux term is simplified by using the Rosseland diffusion approximation for an optically thick fluid according to

$q_{r}=-\frac{4 \sigma}{3 \kappa\left(a+\sigma_{s}\right)} \frac{\partial T^{4}}{\partial y}$.

In the above expression, $\sigma$ is the Stefan-Boltzman constant, $a$ is the Rosseland mean absorption coefficient and $\sigma_{s}$ is the scattering coefficient.

Now, $\psi$ is the stream function that satisfies the continuity equation (1) and is defined by

$u=\frac{\partial \psi}{\partial y}, \quad v=-\frac{\partial \psi}{\partial x}$.

When $\varepsilon \ll<1$, the functions $\psi, T$ and $C$ may be developed in the following forms

$$
\begin{aligned}
& \psi(x, y, t)=\psi_{s}(x, y)+\varepsilon \psi_{1}(x, y) e^{i \omega t}, \\
& T(x, y, t)=T_{s}(x, y)+\varepsilon T_{1}(x, y) e^{i \omega t}, \\
& C(x, y, t)=C_{s}(x, y)+\varepsilon C_{1}(x, y) e^{i \omega t},
\end{aligned}
$$

where only the real part is to be taken as it has physical meaning.

Now, we substitute (7)-(10) into (2)-(4) and equate the coefficients of $\varepsilon^{0}$ that give

$$
\begin{aligned}
& \frac{\partial \psi_{s}}{\partial y} \frac{\partial^{2} \psi_{s}}{\partial x \partial y}-\frac{\partial \psi_{s}}{\partial x} \frac{\partial^{2} \psi_{s}}{\partial y^{2}}=\frac{1}{2} U_{0}^{2} \\
& +v \frac{\partial^{3} \psi_{s}}{\partial y^{3}}+g \beta_{T}\left(T_{s}-T_{\infty}\right) \cos (\pi / 4) \\
& g \beta_{C}\left(C_{s}-C_{\infty}\right) \cos (\pi / 4), \\
& \frac{\partial \psi_{s}}{\partial y} \frac{\partial T_{s}}{\partial x}-\frac{\partial \psi_{s}}{\partial x} \frac{\partial T_{s}}{\partial y}=\alpha \frac{\partial^{2} T_{s}}{\partial y^{2}} \\
& +\frac{16 \alpha \sigma}{3 \kappa}\left(a+\sigma_{s}\right) \frac{\partial}{\partial y}\left(T_{s}^{3} \frac{\partial T_{s}}{\partial y}\right), \\
& \frac{\partial \psi_{s}}{\partial y} \frac{\partial C_{s}}{\partial x}-\frac{\partial \psi_{s}}{\partial x} \frac{\partial C_{s}}{\partial y}=D \frac{\partial^{2} C_{s}}{\partial y^{2}} .
\end{aligned}
$$

For the set of above equations, $\psi_{s}, T_{s}$ and $C_{s}$ represent the steady-state solutions that can be determined by the following functions: 


$$
\begin{aligned}
& \psi_{s}=\left(v U_{0}\right)^{1 / 2} x^{3 / 4} f(\eta), \frac{T_{s}-T_{\infty}}{T_{w}-T_{\infty}}=\theta(\eta), \\
& \frac{C_{s}-C_{\infty}}{C_{w}-C_{\infty}}=\varphi(\eta), \eta=\left(U_{0} / v\right)^{1 / 2} x^{-1 / 4} y .
\end{aligned}
$$

Using (14) into (11)-(13), we obtain

$$
\begin{aligned}
& f^{\prime \prime \prime}+\frac{3}{4} f f^{\prime \prime}-\frac{1}{2}\left(f^{\prime 2}-1\right) \\
& +(1-w) \operatorname{Ri} \theta+w R i \varphi=0, \\
& \frac{1}{\operatorname{Pr}}\left\{1+\frac{4}{3} R_{d}(1+\Delta \theta)^{3}\right\} \theta^{\prime \prime} \\
& +\frac{4 \Delta}{\operatorname{Pr}} R_{d}(1+\Delta \theta)^{3} \theta^{\prime 2}+\frac{3}{4} f \theta^{\prime}=0, \\
& \frac{1}{S c} \varphi^{\prime \prime}+\frac{3}{4} f \varphi^{\prime}=0,
\end{aligned}
$$

with boundary conditions

$$
\begin{aligned}
& f=f^{\prime}=0, \theta=1, \varphi=1 \quad \text { at } \eta=0 \\
& f^{\prime}=1, \theta=0, \varphi=0 \quad \text { as } \eta \rightarrow \infty .
\end{aligned}
$$

Substituting the expressions (7)-(10) into (2)-(4) and equating the coefficients of $\varepsilon$ give equations for timedependent components $\psi_{1}, T_{1}$ and $C_{1}$ as

$$
\begin{aligned}
& i \omega \frac{\partial \psi_{1}}{\partial y}+\frac{\partial \psi_{0}}{\partial y} \frac{\partial^{2} \psi_{1}}{\partial x \partial y}+\frac{\partial \psi_{1}}{\partial y} \frac{\partial^{2} \psi_{s}}{\partial x \partial y} \\
& -\frac{\partial \psi_{s}}{\partial x} \frac{\partial^{2} \psi_{1}}{\partial y^{2}}-\frac{\partial \psi_{1}}{\partial x} \frac{\partial^{2} \psi_{s}}{\partial y^{2}}=i \omega U_{0} x^{1 / 2}+U_{0}^{2} \\
& +v \frac{\partial^{3} \psi_{1}}{\partial y^{3}}+g\left(\beta_{T} T_{1}+\beta_{C} C_{1}\right) \cos (\pi / 4), \\
& i \omega T_{1}+\frac{\partial \psi_{s}}{\partial y} \frac{\partial T_{1}}{\partial x}+\frac{\partial \psi_{1}}{\partial y} \frac{\partial T_{s}}{\partial x} \\
& -\frac{\partial \psi_{s}}{\partial x} \frac{\partial T_{1}}{\partial y}-\frac{\partial \psi_{1}}{\partial x} \frac{\partial T_{s}}{\partial y}=\alpha \frac{\partial^{2} T_{1}}{\partial y^{2}} \\
& \left.+\frac{16 \alpha \sigma}{3 \kappa\left(a+\sigma_{s}\right.}\right) \frac{\partial}{\partial y}\left(T_{s}^{3} \frac{\partial T_{1}}{\partial y}+3 T_{s}^{2} T_{1} \frac{\partial T_{s}}{\partial y}\right) \\
& i \omega C_{1}+\frac{\partial \psi_{s}}{\partial y} \frac{\partial C_{1}}{\partial x}+\frac{\partial \psi_{1}}{\partial y} \frac{\partial C_{s}}{\partial x} \\
& -\frac{\partial \psi_{s}}{\partial x} \frac{\partial C_{1}}{\partial y}-\frac{\partial \psi_{1}}{\partial x} \frac{\partial C_{s}}{\partial y}=D \frac{\partial^{2} C_{1}}{\partial y^{2}} .
\end{aligned}
$$

The associated boundary conditions become

$$
\begin{aligned}
& \psi_{1}=\frac{\partial \psi_{1}}{\partial y}=0, T_{1}=T_{w}-T_{\infty}, \\
& C_{1}=C_{w}-C_{\infty} \text { at } y=0
\end{aligned}
$$

$$
\frac{\partial \psi_{1}}{\partial y}=U_{0} x^{1 / 2}, T_{1}=0, C_{1}=0 \text { as } y \rightarrow \infty .
$$

To non-dimensionalize the equations (20)-(22), we consider the following expressions

$$
\begin{aligned}
& \psi_{1}=\left(v U_{0}\right)^{\frac{1}{2}} x^{\frac{3}{4}} F(\eta), \frac{T_{1}}{T_{w}-T_{\infty}}=\Theta(\eta), \\
& \frac{C_{1}}{C_{w}-C_{\infty}}=\Phi(\eta) .
\end{aligned}
$$

Using (14) and (25) into (20)-(22), we obtain

$$
\begin{aligned}
& F^{\prime \prime \prime}+\frac{3}{4}\left(f F^{\prime \prime}+f^{\prime \prime} F\right)-f^{\prime} F^{\prime}+i \xi\left(1-F^{\prime}\right) \\
& +(1-w) \operatorname{Ri\Theta }+w R i \Phi+1 \\
& =\frac{1}{2} \xi\left(f^{\prime} \frac{\partial F^{\prime}}{\partial \xi}-f^{\prime \prime} \frac{\partial F}{\partial \xi}\right), \\
& \frac{1}{\operatorname{Pr}}\left\{1+\frac{4}{3} R_{d}(1+\Delta \theta)^{3}\right\} \Theta^{\prime \prime} \\
& +\frac{4 \Delta}{\operatorname{Pr}} R_{d}(1+\Delta \theta)^{2}\left(2 \theta^{\prime} \Theta^{\prime}+\theta^{\prime \prime} \Theta\right) \\
& +\frac{8 \Delta^{2}}{\operatorname{Pr}} R_{d}(1+\Delta \theta) \theta^{\prime 2} \Theta+\frac{3}{4}\left(F \theta^{\prime}+f \Theta^{\prime}\right) \\
& -i \xi \Theta=\frac{1}{2} \xi\left(f^{\prime} \frac{\partial \Theta}{\partial \xi}-\theta^{\prime} \frac{\partial F}{\partial \xi}\right), \\
& \frac{1}{S c} \Phi^{\prime \prime}+\frac{3}{4}\left(f \Phi^{\prime}+\theta^{\prime} F\right)-i \xi \Phi \\
& =\frac{1}{2} \xi\left(f^{\prime} \frac{\partial \Phi}{\partial \xi}-\varphi^{\prime} \frac{\partial F}{\partial \xi}\right) .
\end{aligned}
$$

Here $\xi=\omega x^{1 / 2} / U_{0}, G r_{x}=G r_{T}+G r_{C}=g\left\{\beta_{T}\left(T_{w}-T_{\infty}\right)+\beta_{C}\right.$ $\left.\left(C_{w}-C_{\infty}\right)\right\} \cos (\pi / 4) x^{3} / v^{2}$ is the global Grashof number with $G r_{T}$ being the Grashof number for thermal diffusion and $G r_{C}$ the Grashof number for mass diffusion, $R e_{x}=U_{0} x^{3 / 2} / v$ is the Reynolds number, $R i=G r_{x} / R e_{x}^{2}$ is the Richardson's number, $\operatorname{Pr}=v / \alpha$ is the Prandtl number, $S c=v / D$ is the Schmidt number. Other parameters are defined as $R_{d}=4 \sigma$ $T_{\infty}^{3} / \kappa\left(a+\sigma_{s}\right), \Delta=T_{w} / T_{\infty}-1$ where $R_{d}$ is the conductionradiation parameter and $\Delta$ is the surface temperature parameter.

The corresponding boundary conditions for (26)-(28) are

$$
\begin{aligned}
& F=F^{\prime}=0, \Theta=1, \Phi=1 \text { at } \eta=0 \\
& F^{\prime}=1, \Theta=0, \Phi=0 \text { as } \eta \rightarrow \infty .
\end{aligned}
$$

The sets of equations (15)-(19) and (26)-(30) are solved by employing the straight forward finite difference method ${ }^{12}$. Of interest are the skin friction, the rate of heat transfer and the mass transfer which are expressed following Kumari et al. ${ }^{7}$ : 


$$
\begin{aligned}
& C_{f}=\frac{(\mu+\kappa)}{\rho U_{0}^{2} x}\left(\frac{\partial^{2} \psi_{1}}{\partial y^{2}}\right)_{y=0}, \\
& q=-\frac{x k_{c}}{k_{c}\left(T_{w}-T_{\infty}\right)}\left(\frac{\partial T_{1}}{\partial y}\right)_{y=0}, \\
& m=-\frac{x D}{D\left(C_{w}-C_{\infty}\right)}\left(\frac{\partial C_{1}}{\partial y}\right)_{y=0} .
\end{aligned}
$$

In this study, the results are presented in terms of $A_{u} / \xi^{1 / 2}$, $A_{q} / \xi^{1 / 2}$ and $A_{m} / \xi^{1 / 2}$ where

$$
\begin{aligned}
& A_{u}=\left|\operatorname{Re}_{x}^{1 / 2} C_{f} /(1+K)\right|=\sqrt{F_{r}^{\prime \prime 2}+F_{i}^{\prime \prime 2}}, \\
& A_{q}=\left|\operatorname{Re}_{x}^{-1 / 2} q\right|=\sqrt{\Theta_{r}^{\prime 2}+\Theta_{i}^{\prime 2}}, \\
& A_{m}=\left|\operatorname{Re}_{x}^{-1 / 2} m\right|=\sqrt{\Phi_{r}^{\prime 2}+\Phi_{i}^{\prime 2}} .
\end{aligned}
$$

Here the real parts of the transverse velocity gradient, temperature gradient, and mass gradient at the surface are $F_{r}{ }^{\prime \prime}, \Theta_{r}^{\prime}$ and $\Phi_{r}^{\prime}$ respectively, and the imaginary parts of those are $F_{i}{ }^{\prime \prime}, \Theta_{i}{ }^{\prime}$ and $\Phi_{i}{ }^{\prime}$.

\section{Results and Discussion}

In this paper, we examine the effects of the Richardson's number, $R i$, the Schmidt number, $S c$, and the conductionradiation parameter, $R_{d}$, on the amplitudes of skin-friction, heat transfer and mass transfer.

Figures 2(a)-(c) depict the effects of varying the Richardson's number, $R i$, on the amplitudes of the skin friction, the heat transfer and the mass transfer, respectively. From the figure 2(a), it is seen that the amplitude of the skin friction is higher for higher values of Richardson's number, $R i$. But the rate of change of the skin friction from the leading edge to the downstream region is higher for lower $R i$. On the other hand, figures 2(b) and 2(c) show that with an increase of $R i$, the amplitudes of both heat and mass transfer are higher near the leading edge while the reverse case is observed in the downstream region.

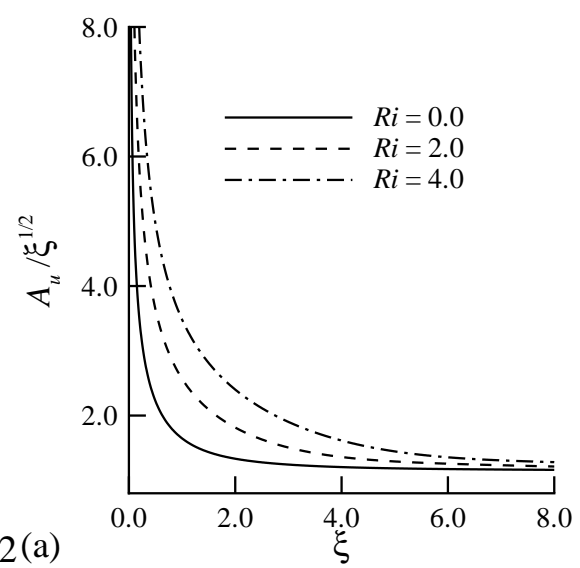

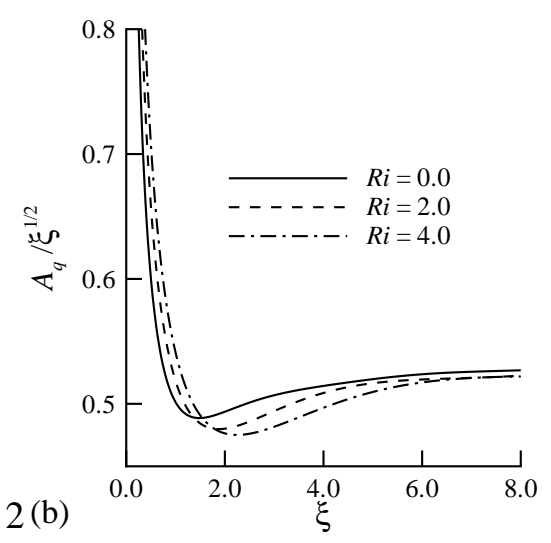

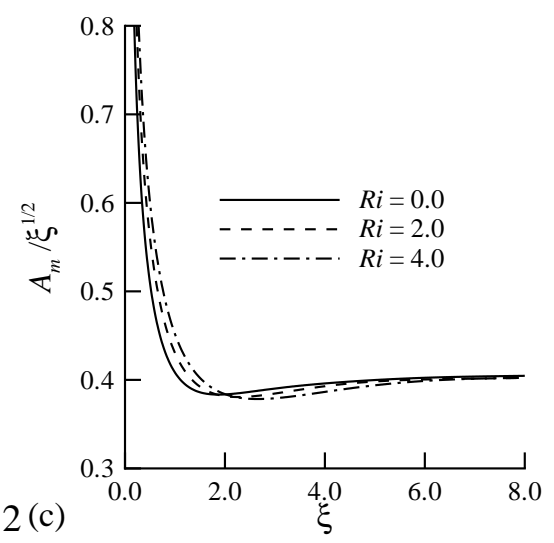

Fig. 2. Amplitudes of (a) skin friction, (b) heat transfer and (c) mass transfer for different values of $R i$ while $\operatorname{Pr}=0.72, w=0.5, S c$ $=0.22, R_{d}=0.5, \Delta=0.1$.

The effects of the Schmidt number, $S c$, on the amplitudes of the skin friction, the heat transfer and the mass transfer are shown in Figures 3(a)-(c), respectively. Results indicate that the amplitude of the skin friction increases with an increase of $S c$. This is due to the fact that the Schmidt number becomes high owing to either increase of the kinematic viscosity or decrease of the mass diffusivity of the fluid, and the increase of the skin friction is the result of this change of the fluid property. The changing of $S c$ has no strong effect on the amplitude of the heat transfer although it has strong effect on the amplitude of the mass transfer. As the value of $S c$ increases, the amplitude of mass transfer increases. It is because the Schmidt number, $S c$, indicates the physical properties of the diffusing species.

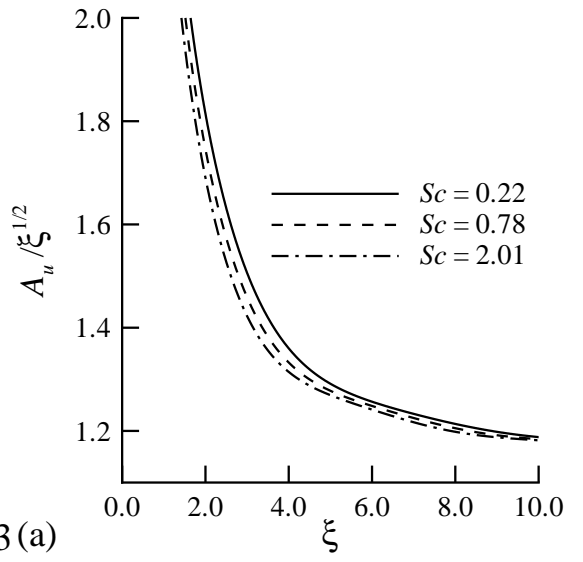



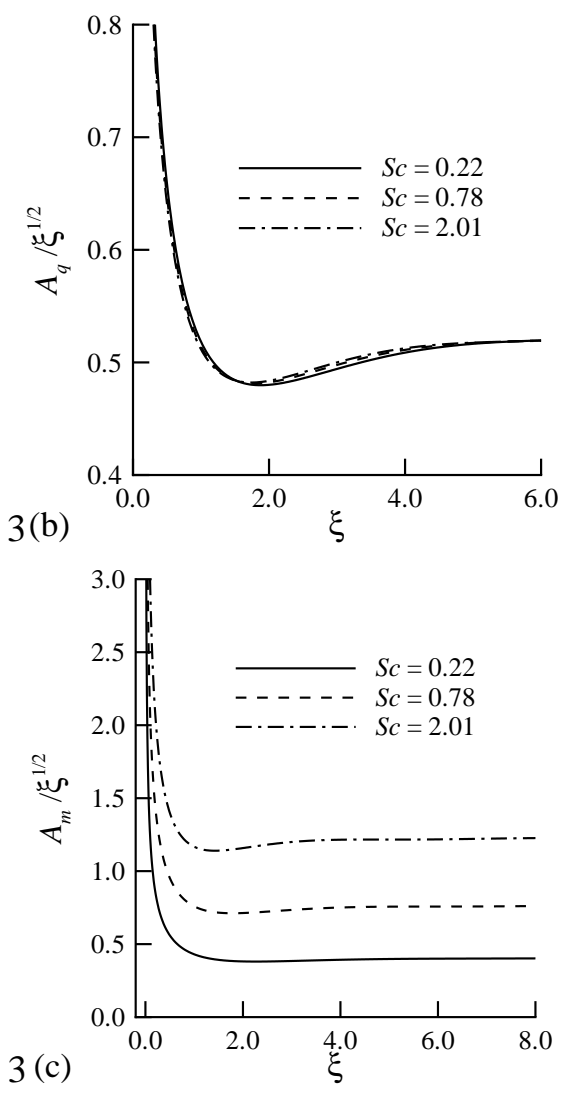

Fig. 3. Amplitudes of (a) skin friction, (b) heat transfer and (c) mass transfer for different values of $S c$ while $\operatorname{Pr}=0.72, R i=2.0, w$ $=0.5, R_{d}=0.5, \Delta=0.5$.

Figures 4(a)-(c) exhibit the influences of the conductionradiation parameter, $R_{d}$, on the amplitudes of the skin friction, the heat transfer and the mass transfer, respectively. From the figures, we observe that the amplitude of the heat transfer is strongly dependent on the conduction-radiation parameter $R_{d}$, whereas that of the skin and the mass transfer is almost unaffected by it. Also an increase in the conduction-radiation parameter results in decreasing the amplitude of the heat transfer which validates the findings of Chamkha et al. ${ }^{10}$. The reason is that the smaller $R_{d}$ corresponds to an increased dominance of conduction over radiation.

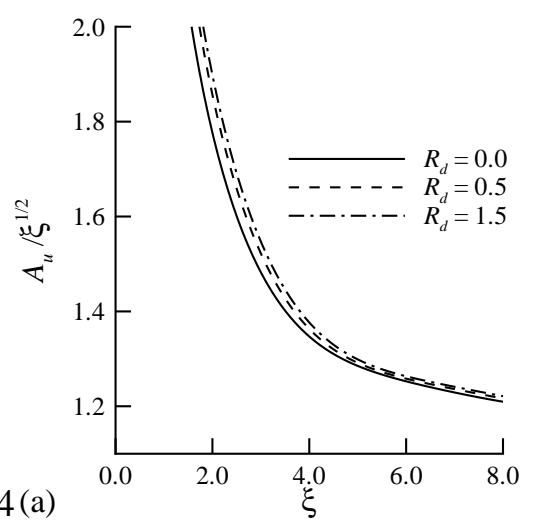

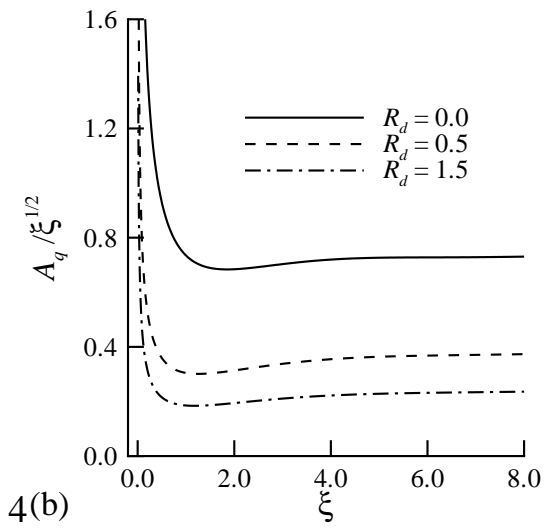

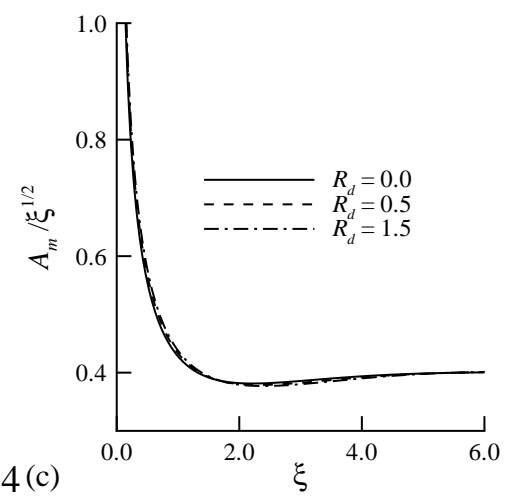

Fig. 4. Amplitudes of (a) skin friction, (b) heat transfer and (c) mass transfer for different values of $R_{d}$ while $\operatorname{Pr}=0.72, R i=2.0, w$ $=0.5, S c=0.22, \Delta=0.5$.

\section{Conclusions}

We study the unsteady mixed convection boundary layer flow over a vertical wedge in the presence of thermal radiation. The dimensionless governing equations have been solved by the straightforward finite difference method for the entire frequency range. We found that for higher Richardson's number, $R i$, the amplitude of the skin friction is higher. With an increase of $R i$, the amplitudes of the heat transfer and the mass transfer are found to be higher near the leading edge but the reverse case is seen in the downstream region. When the Schmidt number, $S c$, is decreased, the amplitude of the skin friction increases. On the contrary, the changing of $S c$ has no strong effect on the amplitude of the heat transfer. Moreover, the amplitude of the mass transfer increases as the value of $S c$ increases. Also the heat transfer is found to be strongly dependent on the conductionradiation parameter, $R_{d}$, but the effect of this parameter on the skin friction and the mass transfer is rather weak.

\section{References}

1. Lighthill, M. J., 1954. The response of laminar skin friction and heat transfer to fluctuations in the stream velocity. Proc. R. Soc., Lond. A., 224, 1-23.

2. Yih K., A., 1998. Uniform suction/blowing effects on forced convection about a wedge. Acta Mech., 128, 173-181.

3. Watanabe, T., 1990. Thermal boundary-layer over a wedge with uniform suction or injection in forced flow. Acta Mech., 83, 119-126. 
4. Sing, P. J., S. Roy, R. Ravindran, 2009. Unsteady mixed convection flow over a vertical wedge, Int. J. Heat Transf., 52, 415-421.

5. Al-Harbi, S. M., and F. S. Ibrahim, 2015. Unsteady mixed convection boundary layer flow along a symmetric wedge with variable surface temperature embedded in a saturated porous medium. Int. J. Num. Methods Heat Fluid Flow, 25, 1162-1175.

6. Prasad, K. V., P. S. Datti, K. Vajravelu, 2013. MHD mixed convection flow over a permeable non-isothermal wedge. J. King Saud Univ., 25, 313-324.

7. Kumari, M., H. S. Takhar and G. Nath, 2001. Mixed convection flow over a vertical wedge embedded in a highly porous medium. Heat Mass Transf., 37, 139-146.

8. Hossain, M. A., M. S. Munir, M. Z. Hafiz and H. S. Takhar, 2000. Flow of a viscous incompressible fluid of temperature dependent viscosity past a permeable wedge with uniform surface heat flux. Heat Mass Transf., 36, 333-341.
9. Kandasamy, R., I. Muhaimin and A. B. Khamis, 2009. Thermophoresis and variable viscosity effects on MHD mixed convective heat and mass transfer past a porous wedge in the presence of chemical reaction, Heat Mass Transf., 45, 703-712.

10. Yih K. A., 2001. Radiation effect on mixed convection over an isothermal wedge in porous media: the entire regime. Heat Transf. Eng., 22, 26-32.

11. Chamkha, A. J., M. Mujtaba, A. Quadri and C. Issa, 2003. Thermal radiation effects on MHD forced convection flow adjacent to a non-isothermal wedge in the presence of heat source or sink. Heat Mass Transf., 39, 305-312.

12. Hossain, M. M., A. C. Mandal, N. C. Roy, and M. A. Hossain, 2013. Fluctuating flow of thermomicropolar fluid past a vertical surface. Applications and Applied Mathematics: An International Journal, 8, 128-150. 
